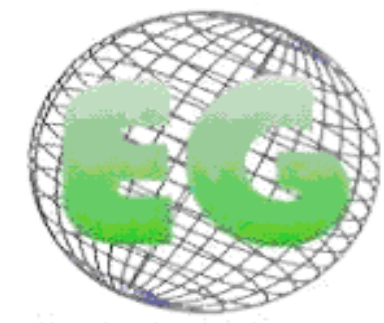

ISSN 1695-6141 $N^{\circ} 26$

\title{
Revisión de los estudios sobre los profesionales de enfermería de las Unidades de Cuidados Intensivos de España
}

\author{
Review of studies on nurses of intensive care of Spain units.
}

\author{
*Navarro Arnedo, Jesús Manuel \\ *Diplomado en Enfermería. Unidad de Cuidados Intensivos. Hospital General Universitario de Alicante. \\ E-mail:Navarro_jes@gva.es
}

Palabras clave: enfermería; unidad de cuidados intensivos; producción científica

Keywords: : nursing; intensive care unit; scientific production.

\section{RESUMEN}

Con el objetivo de analizar la producción científica sobre las enfermeras de UCI en España, se realizó una búsqueda bibliográfica de los artículos en que éstas fueran el sujeto a estudio. Se analizaron 76 artículos, el $64 \%$ escritos por enfermeras de UCl y el $44 \%$ publicados en Enfermería Intensiva. Los estudios que describían a los profesionales y su trabajo eran los más numerosos mientras sobre temas éticos e informáticos eran los más escasos. Aunque entre el $39 \%$ y el $61 \%$ de las enfermeras habían elegido voluntariamente $\mathrm{UCl}$, la mayoría (55\%-82\%) no se trasladarían a otro servicio. Las enfermeras afirmaban que su trabajo era estresante debido al estado de los pacientes y al entorno laboral. Dos trabajos encontraron niveles altos de burnout en enfermeras y auxiliares y en otros siete eran medios o bajos. Las enfermeras opinaban, tras modificar las normas de visita, que la familia proporcionaba apoyo emocional al paciente, minimizaba su aburrimiento e incrementaba su deseo de vivir. Varios estudios concluían que era necesaria preparación específica para enfrentarse a la muerte y dar apoyo al paciente y su familia. El 94\% de las enfermeras opinaban que se debía informar al paciente y familia de su situación y posibilidades y, el $98 \%$, que la familia debía recibir la misma información que el paciente. La mayoría de las aplicaciones informáticas incorporadas en las unidades de cuidados intensivos recibieron evaluaciones positivas de sus usuarias; las enfermeras utilizaban internet para enviar y recibir correo electrónico y buscar información sobre salud.

\section{ABSTRACT}

In order to analyse the scientific output of ICU nurses in Spain, a literature review of articles was carried out in which ICU nurses were the subject under study 76 articles were analyzed, were $64 \%$ written by ICU nurses and $44 \%$ were published in Enfermeria Intensiva. Studies describing the professionals and their work were the most numerous, while those that focused on ethical and computer issues were the most scant. Although between $39 \%-61 \%$ of nurses said they had chosen ICU voluntarily, most of the nurses (55\%-82\%) would not leave for another service. Nurses stated that their work was stressful because of the state of patients and the work environment. Two works found high burnout levels in nurses and nurses' assistants and in another seven these 
were repored as middle or low. After modifying the policy of family visits, nurses affirm that the family gave emotional support to the patient, minimised boredom and increased the desire of live. Several studies concluded that it was necessary specific training to confront death and to give support to the patient and the family. $94 \%$ of nurses thought the patient and the family should be kept informed regarding the former's situation and possibilities and $98 \%$ that the family should receive the same information as the patient. Most of the computer applications incorporated in intensive care units received positive evaluations by users; nurses used Internet to send and receive emails and to seek health information.

\section{INTRODUCCIÓN}

La primera Unidad de Cuidados Intensivos (en adelante UCl) se inauguró en España en 1965; progresivamente, y con un período de máxima expansión entre 1970 y $1990^{(1,2)}$, los hospitales se fueron dotando de estas unidades y servicios, siendo actualmente unas 275 las $\mathrm{UCl}$ en funcionamiento con aproximadamente 3.500 camas instaladas (una media de 12 camas por servicio). A lo largo de estos más de 40 años, las enfermeras de cuidados intensivos (en el artículo se hace referencia a la/s enfermera/s para referirse a los profesionales de ambos géneros) han publicado, en las diferentes revistas científicas de nuestro país, numerosos trabajos de investigación que han sido estudiados por diversos autores, tanto en análisis bibliométricos (determinando autores, temas, procedencia, referencias bibliográficas.... ${ }^{(3-8)}$ como en revisiones bibliográficas sobre un tema concreto ${ }^{(9-}$ 13). Una parte de esta producción científica la conforman investigaciones en las que los sujetos a estudio son las propias enfermeras de $\mathrm{UCl}$, pero no se ha encontrado en la literatura ninguna revisión que analice estos estudios. Así pues, los objetivos de este trabajo son describir el estado de la producción científica sobre las enfermeras de UCI en España y analizar su contenido.

\section{MATERIAL Y MÉTODOS}

Se diseñó un estudio de revisión de los artículos publicados en revistas científicas españolas (tanto en papel como en formato digital) hasta diciembre de 2009, en los cuales las enfermeras de cuidados intensivos (tanto de adultos como pediátricas) fueran el sujeto a estudio; se excluyeron artículos no originales, revisiones críticas, proyectos de investigación y comunicaciones a congresos. Los trabajos que incluían a otros participantes (otros miembros del equipo de $\mathrm{UCl}$, estudiantes, familiares, etc.) se analizaron si las enfermeras representaban más del $50 \%$ de la muestra o los resultados correspondientes a éstas se exponían claramente por separado. La búsqueda bibliográfica se realizó en Medline (limitando la búsqueda a las publicaciones españolas), Cuiden Plus, Cantarida y Enfispro, utilizando las palabras claves "enfermera", "enfermería", "unidad de cuidados intensivos", "críticos" y "cuidados críticos" (en sus acepciones en español e inglés) y el operador "AND" como enlace y se amplió con la búsqueda cruzada de artículos reseñados en la bibliografía; tras depurar los resultados se procedió a la recuperación física de los ejemplares.

De cada artículo se recogió su información bibliográfica (autores, revista, año de publicación, tipo de trabajo, metodología utilizada y tema estudiado según las palabras claves si estaban presentes o el tema principal del trabajo) y los resultados y conclusiones más relevantes. Los datos se muestran en cifras absolutas y porcentajes, para la obtención de los cuales se utilizo el programa Epiinfo 6.0.

\section{RESULTADOS}

Setenta y seis artículos cumplieron los criterios de inclusión ${ }^{(14-89)}$, el $64 \%$ escritos por enfermeras que trabajaban mayoritariamente (82\%) en hospitales. En 39 trabajos (51\%) 
participaron sólo enfermeras y en 26 (34\%) enfermeras y auxiliares de enfermería; las características de los estudios se muestran en la tabla I.

Tabla I. Características de los autores y de los trabajos analizados.

\begin{tabular}{|c|c|c|}
\hline & $\begin{array}{c}\mathrm{n}^{\mathrm{o}} \\
(\mathrm{n}=76)\end{array}$ & $\%$ \\
\hline \multicolumn{3}{|l|}{ Número de autores: } \\
\hline Uno: & 5 & $6 ’ 6$ \\
\hline Dos. & 13 & 17 '1 \\
\hline Tres: & 17 & $22{ }^{\prime} 4$ \\
\hline Cuatro: & 16 & $21 ' 1$ \\
\hline Cinco: & 11 & $14^{\prime} 5$ \\
\hline Seis o más: & 14 & $18^{\prime} 4$ \\
\hline \multicolumn{3}{|l|}{ Sexo: } \\
\hline Mujer: & 40 & 57 \\
\hline Hombre: & 30 & 43 \\
\hline No se puede deducir de los datos del artículo: & 6 & 10 \\
\hline \multicolumn{3}{|l|}{ Puesto de trabajo del primer autor: } \\
\hline Enfermera/o: & 49 & $64^{\prime} 5$ \\
\hline Profesor/a universidad: & 11 & $14^{\prime} 5$ \\
\hline Intensivista: & 8 & $10^{\prime} 5$ \\
\hline Supervisora/or: & 5 & $6 \cdot 6$ \\
\hline Otro: & 3 & $3 ’ 9$ \\
\hline
\end{tabular}

Centro de trabajo del primer autor:

$\begin{array}{rcc}\text { Hospital: } & 62 & 81^{\prime} 6 \\ \text { Universidad: } & 11 & 14^{\prime} 5 \\ \text { Otros: } & 3 & 3,9\end{array}$

Profesionales participantes en el estudio:

Enfermeras: $\quad 39 \quad 51$ '3

Enfermeras y auxiliares de enfermería: $\quad 26 \quad 34$ '2

Enfermeras e intensivistas: 4 5'3

Enfermeras, auxiliares de enfermería e intensivistas: $\quad 3 \quad 3$ '9

Enfermeras y familiares, pacientes, estudiantes...: 4 5'3 Sección:

Originales: $\quad 68 \quad 89^{\prime} 5$

Original breve: $\quad 3 \quad 3$ '9

Diseño:

Otra (cartas al director, artículo especial,...): $5 \quad$ 6'6

Metodología:

Descriptivo: $\quad 71 \quad 93$ '4

Prospectivo: $\quad 5 \quad 6$ '6

Cuantitativa: $\quad 65 \quad 85^{\prime} 5$

Cualitativa: $11 \quad 14$ '5

Estudio financiado/becado:

Si: $\quad 8 \quad 10^{\prime} 5$

No: $68 \quad 89^{\circ} 5$ 
La revista Enfermería Intensiva publicó el 44\% de los mismos (figura 1).

Figura 1. Revistas de publicación.

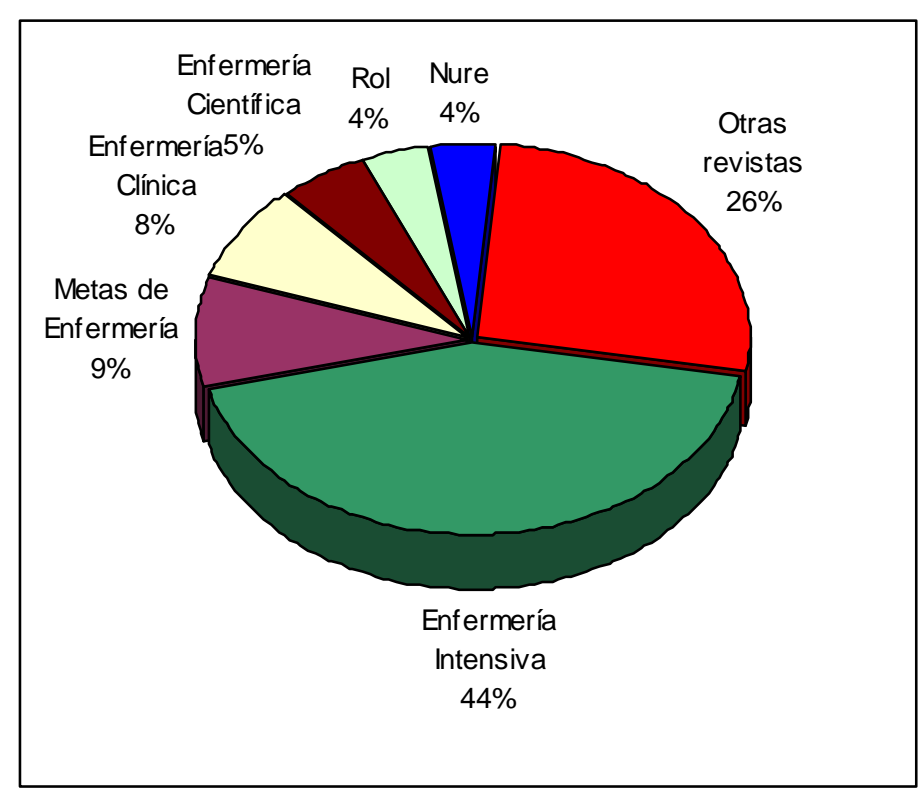

El artículo más antiguo databa de 1975, 2002 era el año en que más trabajos se editaron (9/12\%) mientras en 1997 no se publicó ninguno (figura 2).

Figura 2. Años de publicación de los estudios

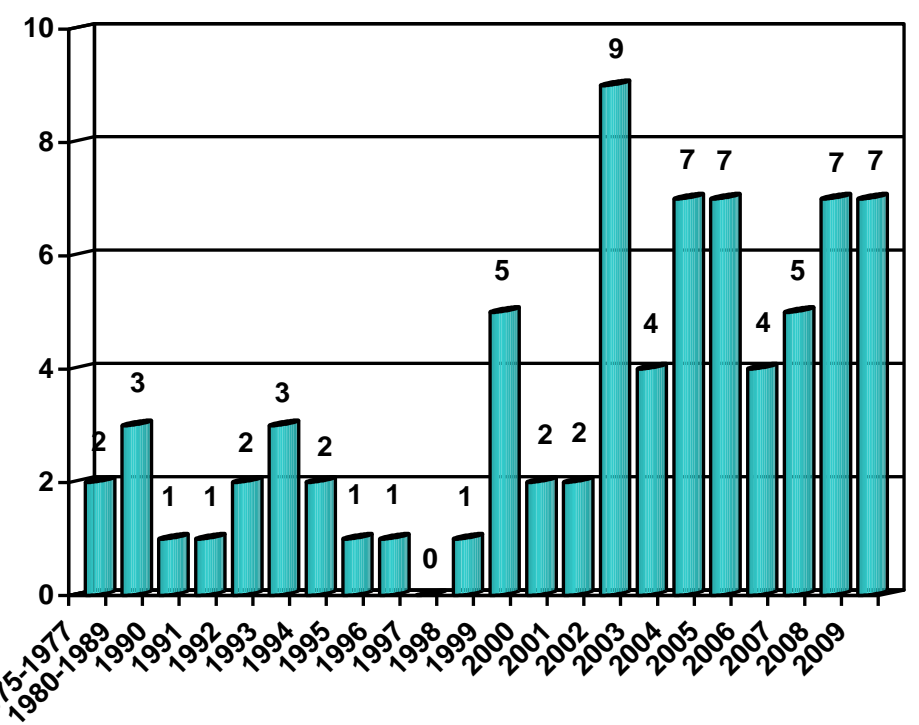

Las comunidades autónomas que aportaban un mayor número de investigaciones fueron Madrid (21/28\%), Cataluña (12/16\%) y Comunidad Valenciana (10/13\%) (figura 3). 
Figura 3. Comunidad Autónoma de procedencia de los trabajos analizados

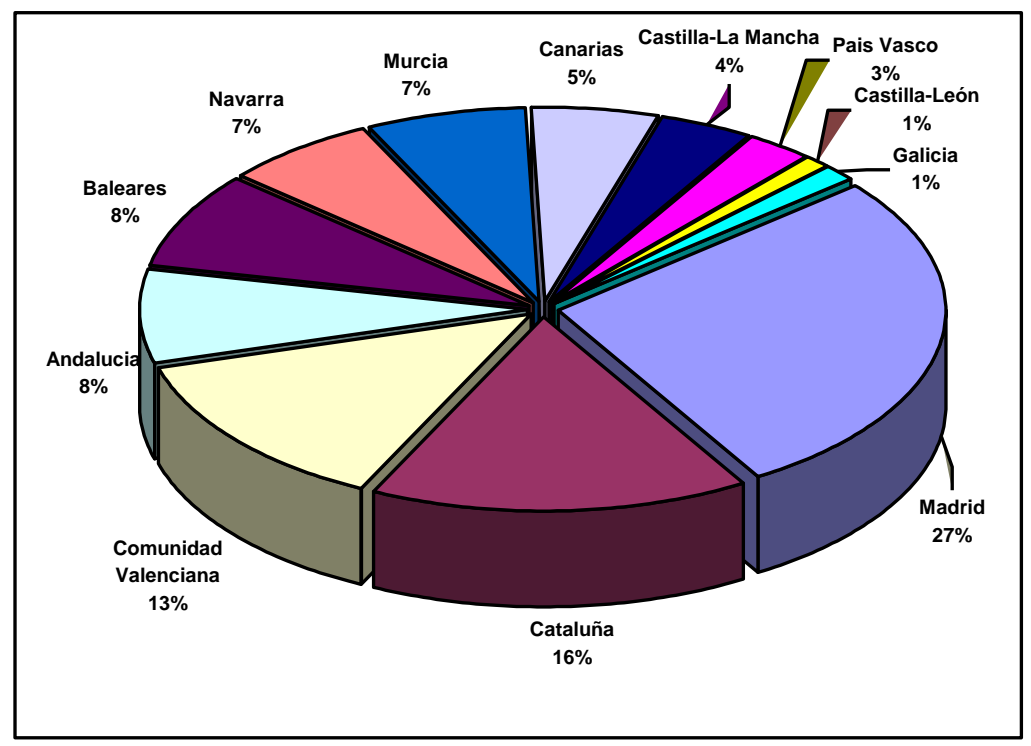

Los artículos se clasificaron según el tema del estudio en 8 categorías, siendo los que describían a los profesionales (perfil profesional) y su trabajo en las Unidades de Cuidados Intensivos los más numerosos; las 8 categorías se pueden consultar en la tabla II.

Tabla II.- Tema principal de los trabajos analizados.

\begin{tabular}{|c|c|c|}
\hline & $\begin{array}{c}\mathrm{n}^{\mathrm{o}} \\
(\mathrm{n}=76)\end{array}$ & $\%$ \\
\hline Los profesionales de/en las UCI: & 25 & $32 ’ 9$ \\
\hline Clima laboral/condiciones de trabajo/plantillas: & 11 & \\
\hline Descripción/perfil: & 9 & \\
\hline Inicios en UCI/formación: & 3 & \\
\hline Enfermeras como pacientes: & 2 & \\
\hline Síndrome de burnout: & 12 & $15^{\prime} 8$ \\
\hline Evaluación de técnicas y/o conocimientos: & 9 & $11^{\prime} 8$ \\
\hline Familiares: & 9 & $11 ' 8$ \\
\hline Estrés: & 8 & $10 ’ 5$ \\
\hline Muerte: & 5 & $6{ }^{\prime} 6$ \\
\hline Informática: & 4 & $5 \cdot 3$ \\
\hline Ética: & 4 & 5,3 \\
\hline
\end{tabular}

\section{Los profesionales y su trabajo en las Unidades de Cuidados Intensivos}

Los profesionales de enfermería de UCl. De los estudios que ofrecían un perfil de las enfermeras de $\mathrm{UCI}^{(14-23)}$, se obtuvo que la mayoría $\left(87 \%{ }^{(16)}\right.$ al $\left.99 \%{ }^{(14)}\right)$ eran mujeres de 25 a 40 años ${ }^{(14,16)}$, con plaza en propiedad y 5 a 10 años de experiencia en $\mathrm{UCI}^{(16,17)}$, servicio que había elegido entre el $39 \%^{(20)}$ y el $61 \%^{(17,18,23)}$, que no conllevaba incentivo alguno ${ }^{(16)}$ y del que no deseaba trasladarse entre el $55 \%$ y el $82 \%^{(17-21,23-25)}$. Ninguna enfermera creía poder 
aguantar muy bien toda su vida profesional en $\mathrm{UCI}^{(18)}$, opinando el $78 \%$ que debería poder salir de la unidad al solicitarlo( ${ }^{(18)}$; en el estudio de Salas ${ }^{(25)}$, el $85 \%$ de las enfermeras proponía establecer un periodo máximo de permanencia en intensivos. Francisco ${ }^{(14)}$ y Esteban ${ }^{(26)}$ comprobaron que el mayor índice de traslados se producía a los dos años de estar en UCl, superados los cuales muchas profesionales permanecían más de 5 años en intensivos $^{(14)}$; tras más de 10 años en $\mathrm{UCl}$, el $67 \%$ de las enfermeras ${ }^{(23)}$ se habían trasladado a otro servicio por trabajar en un turno fijo.

Los valores identificados por las enfermeras al atender a los enfermos se circunscribían al paradigma humanista (respeto a la condición humana y/o la sensibilidad hacia el sufrimiento que conlleva la experiencia de enfermar) ${ }^{(27)}$.

En cuanto al personal auxiliar de enfermería, según el trabajo de Cuenca ${ }^{(16)}$ el 99'7\% eran mujeres, con una antigüedad media en el servicio de 6'5 años y plaza en propiedad el 81 '6\%; aunque Francisco ${ }^{(14)}$ encontró grandes diferencias en su distribución (de 2'5 a 14 camas por auxiliar), estudios posteriores determinaron que, en el $73 \%$ de UCI, cada auxiliar atendía 3 ó 4 camas $^{(15)}$, Cuenca ${ }^{(16)}$ encontró una relación auxiliar/cama de 1 '17 y Torné ${ }^{(28)}$ de $1 ' 28$.

Formación de las enfermeras. Aunque en 1995 el 42'6\% de las $\mathrm{UCl}^{(16)}$ contaba con programas de formación para enfermeras de nueva incorporación, el $67 \%$ de los profesionales opinaba, en un estudio en $2003^{(28)}$, que la incorporación de personal nuevo suponía un gran problema. Por su parte, las enfermeras recién llegadas a UCI consideraron que, al inicio de su trabajo, era fundamental una buena predisposición ${ }^{(18,29)}$ y afirmaron haber aprendido de los compañeros ${ }^{(20,29)}$ y de su experiencia diaria en la unidad ${ }^{(20)}$, el trabajo en equipo era lo que más les había gustado ${ }^{(29,30)}$ y la tecnología lo que les había supuesto la principal dificultad al adaptarse ${ }^{(25,29,30)}$.

La formación continuada en UCI ha sido abordada desde diversos ángulos. Respecto a la oferta de formación, si en 1980 la mitad de las unidades organizaba estas actividades al menos 1 vez al año ${ }^{(14)}$, en 1994 las UCl ofrecían una media de 2'3 cursos anuales ${ }^{(15)}$. En cuanto a la opinión de las enfermeras, el $99 \%$ creía ${ }^{(14,17,18,20,25,29)}$ que la formación, tanto previa a trabajar en UCI como continuada, era imprescindible, afirmando el $73 \%$ de las mismas que eran necesarios de 2 a 3 años de ejercicio profesional para conseguir dominio y seguridad en el trabajo ${ }^{(17,19)}$. La visión de los formadores la ofreció el estudio de Palacín, realizado tras ocho años impartiendo un curso de posgrado de enfermería intensiva ${ }^{(31)}$ : al $97 \%$ de los alumnos el curso les había servido para tener mucha o bastante seguridad, fiabilidad y confianza en su trabajo en $\mathrm{UCl}$ y los profesionales responsables de los alumnos afirmaban que la sobrecarga de trabajo físico e intelectual que suponían éstos quedaba compensada durante el período de prácticas.

El $99 \%$ de las enfermeras en varios estudios creía necesaria la especialidad en cuidados intensivos ${ }^{(14-18)}$.

Investigación. En 1995, sólo el 6\% ${ }^{(16)}$ de las UCl realizaba actividades de investigación de forma habitual y el $93 \%$ de forma esporádica, la media de asistencias a congresos nacionales era de 2'3, a congresos internacionales de 0'1 y el número publicaciones por año de 0 ' $6^{(15)}$.

El trabajo en UCl. Francisco ${ }^{(14)}$, en 1980, encontró que cada enfermera cuidaba de 3 ó 4 pacientes, cifra que pasó a 1 enfermera por cada 2-3 enfermos en estudios posteriores $^{(15,16,28,32)}$ (ratio enfermera/paciente de 2'18-2'214), si bien Torné(28) ${ }^{\text {concluyó que }}$ 
la plantilla era insuficiente en el $56 \%$ de las UCl estudiadas y propuso formulas de cálculo de ésta basadas en la complejidad de la unidad.

En la mayoría de servicios (53-86\%) el trabajo se desarrollaba a turno rodado ${ }^{(16,19,20)}$, considerando las enfermeras ${ }^{(21)}$ que su trabajo las obligaba a permanecer de pie, hacer esfuerzos considerables, mantener una gran concentración y, ocasionalmente, asumir tareas que no les correspondían por su categoría profesional ${ }^{(21)}$, resultado que también obtuvo Lozano $^{(15)}$ al determinar que el $95 \%$ de UCl no contaba con otros especialistas (fisioterapeutas, sicólogos,...) y su trabajo lo asumían las enfermeras.

Las enfermeras describieron las UCl como servicios con mucho ruido ${ }^{(21,33)}$ en los que realizaban técnicas y cuidados específicos y exclusivos de cuidados intensivos ${ }^{(15)}$, similares en todas las unidades pero protocolizados sólo en el $36 \%$ de éstas ${ }^{(15)}$; Maull ${ }^{(32)}$, en una investigación en $13 \mathrm{UCl}$ de Cataluña, encontró que el $61 \%$ de las rutinas no estaban basadas en estudios científicos y se hacían por inercia.

En el $44 \%$ de unidades de cuidados intensivos ${ }^{(15)}$ se realizaban sesiones de enfermería y sólo en el 34\% las enfermeras participaban en las sesiones médicas; sin embargo, la valoración conjunta y consensuada de los enfermos, según el estudio de Ventura ${ }^{(34)}$, producía en enfermería una mejora en todas las dimensiones de satisfacción, expectativas y desempeño profesional.

El $76 \%$ de las enfermeras no consideró su trabajo monótono o aburrido y, entre el $79 \%$ y el $85 \%$ se mostró satisfecha con el mismo ${ }^{(19,21)}$; el motivo de satisfacción más enunciado era el trabajo en equipo ${ }^{(21,23,30,35,37,38)}$, al que se concede tanta relevancia que, para el $87 \%$ de las enfermeras del estudio de Díaz ${ }^{(18)}$, lo peor que le puede pasar a una unidad es que el equipo falle, y que precisa de una buena comunicación interdisciplinaria y del respeto y reconocimiento mutuo de cada uno de sus miembros y de su trabajo ${ }^{(27)}$; García ${ }^{(24)}$ determinó que cuando las enfermeras tenían más pacientes a su cargo, las relaciones en el equipo eran insatisfactorias. El salario constituía el principal motivo de insatisfacción/problemática más frecuente (tabla III).

Tabla III.- Motivos de satisfacción, insatisfacción y problemas en UCI.

Motivos de satisfacción:

$$
\begin{array}{r}
\text { Trabajo en equipo }^{(21,23,30,35,37,38)} . \\
\text { Relacion con el paciente }^{(20,23,30,35)} . \\
\text { Relación con el superior inmediato }{ }^{(21,23)} . \\
\text { Reconocimiento del trabajo realizado } \\
\text { Condiciones físicas del trabajo }
\end{array}
$$

Motivos de insatisfacción:

$$
\begin{array}{r}
\text { Salario }^{(18-21,36,37)} . \\
\text { Pocas posibilidades de promoción }{ }^{(19,21,37)} . \\
\text { Trabajo a turnos }^{(18,20)} . \\
\text { Presión asistencial }^{(37)} . \\
\text { Modo en que está gestionada la empresa } \\
\\
\text { Monotonía laboral }^{(37)} .
\end{array}
$$


Problemática:

Poca remuneración económica ${ }^{(16,18-21,25,37)}$.
Trabajo a turnos ${ }^{(18,20,22)}$.
Formación inadecuada ${ }^{(16,19,20,22)}$.
Escasez de recursos humanos ${ }^{(16,21,28)}$.
Falta de promoción profesional ${ }^{(21,37)}$.
Falta de motivación $^{(16)}$.

Aunque al $61 \%$ de los encuestados por Gámez $^{(21)}$ el trabajo en UCI les había repercutido a veces negativamente en su vida familiar, en otro estudio ${ }^{(23)}$ este porcentaje era sólo del $28 \%$ y el $75 \%$ afirmaban que el trabajo en intensivos le había influido positivamente en su forma de ser al valorar más lo que tenían ${ }^{(23)}$. Esteban ${ }^{(26,39)}$ analizó la personalidad de un grupo de enfermeras mediante el test CEP en dos estudios y encontró que el trabajo en UCl no parecía condicionar una involución de la misma, por lo que era posible que tan sólo una personalidad característica admitiera la permanencia en UCI durante periodos prolongados.

El $74 \%$ de las enfermeras había sufrido agresiones ${ }^{(40)}$, el $68 \%$ verbales (el $58 \%$ por parte de los pacientes) y el $62 \%$ físicas (el $73 \%$ de los pacientes y el $26 \%$ de sus familiares).

Enfermeras como pacientes de UCI. Las enfermeras que habían vivido la experiencia de ingresar en $\mathrm{UCl}$ afirmaron haber sentido miedo (a no saber si iban a morir ${ }^{(41)}$ ) y al salir de la $\mathrm{UCI}^{(42)}$ desorientación (desconocían por qué estaban allí y no podían comunicarse por las medidas invasivas respiratorias) y cansancio $^{(41)}$, valorando el soporte familiar como muy importante ${ }^{(41,42)}$.

\section{El estrés de las enfermeras en las Unidades de Cuidados Intensivos.}

El estrés es una respuesta fisiológica, psicológica y de comportamiento del individuo que intenta adaptarse a presiones internas y externas ${ }^{(43)}$; si éste no puede hacer frente a las demandas físicas y psicológicas que percibe y el estado de estrés se mantiene en el tiempo, se pueden producir efectos nocivos sobre la salud, apareciendo síntomas psicológicos, físicos o conductuales ${ }^{(36,44)}$.

Entre el $54 \%{ }^{(18,19)}$ y el $87 \%{ }^{(23)}$ de las enfermeras afirmaban que su trabajo era estresante; aunque los estudios encontrados utilizaban diferentes escalas para medir el estrés de los profesionales (Nursing Stress Scale ${ }^{(36,45)}$, inventario ISRA ${ }^{(48)}, \ldots$ ), todos identificaron una serie de factores responsables que se pueden clasificar en relacionados con el estado de los pacientes (gravedad y cuidados que necesitan ${ }^{(23,43)}$, realizarles cuidados dolorosos ${ }^{(36,43,45)}$, verlos sufrir $^{(36,44,45)}$ e impotencia ante un paciente que no mejora ${ }^{(36,45)}$ ) y relativos al entorno laboral (escasez de personal ${ }^{(43,45,46)}$, mobiliario incómodo ${ }^{(43)}$, insuficientes recursos materiales ${ }^{(46)}$, problemas en el equipo de $\mathrm{UCI}^{(23,45,46)}$, situaciones de urgencia $^{(19,23)} \mathrm{y}^{\text {temor }}$ tanto a confundir la medicación como a hacer mal el trabajo y perjudicar al paciente ${ }^{(45,47)}$ ).

Los síntomas atribuibles al estrés que presentaban las enfermeras eran irritabilidad ${ }^{(19,43,46,48)}$, ansiedad/nerviosismo ${ }^{(19,43,46,48)}$, trastornos digestivos ${ }^{(19,43)}$, sensación de impotencia ${ }^{(46,48)}$ 'y tristeza ${ }^{(46)}$. 
En el estudio de Simón ${ }^{(36)}$, las profesionales más jóvenes presentaron mayor vulnerabilidad al estrés, posiblemente porque, a mayor edad y antigüedad en la unidad, la enfermera alcanza mayor seguridad en sí misma, crea sus propios mecanismos para regular el estrés y se hace menos vulnerable ${ }^{(36)}$.

Simarro ${ }^{(47)}$ investigó la relación entre estrés y estilos de aprendizaje en su unidad, encontrando que existía una relación de mayor estrés cuando los profesionales tenían niveles bajos de estilo de aprendizaje activo y niveles altos de los estilos de aprendizaje reflexivo y teórico, concluyendo que no actuar de forma arriesgada ni improvisada, sino con reflexión y consideración del riesgo, suponía una mayor percepción de estrés ante las situaciones complicadas.

Las enfermeras, para luchar contra el estrés en sus unidades, actuarían sobre la escasez de personal, el ruido excesivo y los turnos de trabajo ${ }^{(43)}$, establecerían un periodo máximo de permanencia en UCl y facilitarían el traslado a otros servicios ${ }^{(25)}$.

Tomás ${ }^{(45)}$ encontró los mismos niveles de estrés en las enfermeras de cuidados intensivos, urgencias, oncología, cuidados paliativos y hospitalización general, siendo los principales estresores la presión laboral/carga de trabajo y los aspectos relativos a la muerte y al proceso de morir; asimismo García ${ }^{(43)}$ obtuvo el mismo resultado al comparar el estrés en las enfermeras de $\mathrm{UCl}$ y urgencias.

\section{Síndrome de Burnout o estar quemado y cuidados intensivos.}

Cristina Maslach, en 1976, definió el Burnout como un síndrome caracterizado por agotamiento emocional (sensación de sobreesfuerzo físico consecuencia del contacto con las personas a las que hay que atender), despersonalización (falta de sentimientos e insensibilidad de los profesionales) y reducida realización personal (declive en el sentimiento de competencia y éxito en el trabajo) ${ }^{(24,49-53)}$ que aparece en las profesiones que ayudan a los demás (enfermeras, médicos, policia,...).

Los estudios investigando el síndrome de Burnout en las enfermeras de UCl utilizaron el Maslach Burnout Inventory como instrumento de medida, cuestionario de 22 ítems que diferencia las tres dimensiones que conforman el síndrome: agotamiento o cansancio emocional, despersonalización y realización personal en el trabajo. Una puntuación alta en cansancio emocional y despersonalización y una puntuación baja en realización personal reflejan un grado alto de burnout.

Dos autoras encontraron niveles altos de burnout en enfermeras y auxiliares de enfermería: el $16 \%$ de las enfermeras y el $10 \%$ de auxiliares encuestadas por Frade ${ }^{(54)}$ y el $12 \%$ en el estudio de $\operatorname{Rios}^{(53)}$ puntuaban alto en cansancio emocional y despersonalización y bajo en realización personal. En el resto de estudios, los niveles fueron medios ${ }^{(24,50,55-57)}$ o bajos ${ }^{(49,52)}$ (tabla IV). 
Tabla IV.- Estudios sobre el burnout en UCI.

\begin{tabular}{|c|c|c|c|c|}
\hline $\begin{array}{c}\text { Autor y año del } \\
\text { estudio. }\end{array}$ & $\begin{array}{l}\text { Profesionales } \\
\text { incluidos. }\end{array}$ & $\begin{array}{c}\text { Cansancio } \\
\text { emocional. } \\
\text { (media y d.e.) }\end{array}$ & $\begin{array}{c}\text { Despersonalizació } \\
\text { n. } \\
\text { (media y d.e.) }\end{array}$ & $\begin{array}{l}\text { Realización } \\
\text { personal. } \\
\text { (media y d.e.) }\end{array}$ \\
\hline Frade $^{(54)}$ & 123 enfermeras & $21 \pm 11$ & $10 \pm 4$ & $35 \pm 8$ \\
\hline 2009 & $\begin{array}{l}58 \text { aux.enferm. } \\
16 \text { médicos. } \\
14 \text { residentes. }\end{array}$ & $\begin{array}{c}37 \% \text { niveles } \\
\text { elevados. }\end{array}$ & $\begin{array}{l}57 \% \text { niveles } \\
\text { elevados. }\end{array}$ & $\begin{array}{c}37 \% \text { niveles } \\
\text { bajos. }\end{array}$ \\
\hline $\operatorname{Rios}^{(58)}$ & 33 enfermeras. & $25^{\prime} 45 \pm 11^{\prime} 1$ & $8^{\prime} 1 \pm 5^{\prime} 2$ & $36^{\prime} 88 \pm 6^{\prime} 42$ \\
\hline 2008 & 9 aux. enferm. & $\begin{array}{c}54 ' 8 \% \text { niveles } \\
\text { elevados. }\end{array}$ & $\begin{array}{c}35,7 \% \text { niveles } \\
\text { elevados. }\end{array}$ & $\begin{array}{c}21^{\prime} 4 \% \text { niveles } \\
\text { bajos. }\end{array}$ \\
\hline García ${ }^{(24)}$ & 280 enfermeras. & $25^{\prime} 7 \pm 9^{\prime} 8$ & $9^{\prime} 4 \pm 4^{\prime} 2$ & $35^{\prime} 5 \pm 7{ }^{\prime} 5$ \\
\hline 2004 & 86 aux. enferm. & $\begin{array}{c}42 \text { ' } 4 \% \text { niveles } \\
\text { elevados. }\end{array}$ & $\begin{array}{c}44^{\prime} 2 \% \text { niveles } \\
\text { elevados. }\end{array}$ & $\begin{array}{c}43^{\prime} 5 \% \text { niveles } \\
\text { bajos. }\end{array}$ \\
\hline $\begin{array}{c}\text { Carmona }^{(55)} \\
2002\end{array}$ & $\begin{array}{l}29 \text { enfermeras. } \\
18 \text { aux. enferm. }\end{array}$ & $21 \pm 9^{\prime} 1$ & $5^{\prime} 5 \pm 4^{\prime} 52$ & $34^{\prime} 7 \pm 6^{\prime} 48$ \\
\hline $\begin{array}{l}\text { Santana }^{(57)} \\
2009\end{array}$ & $\begin{array}{l}56 \text { enfermeras. } \\
41 \text { aux. enferm. }\end{array}$ & $25^{\prime} 01 \pm 7^{\prime} 24$ & $13^{\prime} 78 \pm$ 4’95 & $22^{\prime} 14 \pm$ 4’94 \\
\hline Solano ${ }^{(49)}$ & 83 enfermeras. & $14^{\prime} 24 \pm 8 ’ 17$ & 4'05 \pm 4'09 & $36^{\prime} 76 \pm 6^{\prime} 71$ \\
\hline 2002 & & $\begin{array}{c}14{ }^{\prime} 5 \% \text { niveles } \\
\text { elevados. }\end{array}$ & $\begin{array}{c}10 \text { '8\% niveles } \\
\text { elevados. }\end{array}$ & $\begin{array}{c}37^{\prime} 3 \% \text { niveles } \\
\text { bajos. }\end{array}$ \\
\hline $\begin{array}{c}\text { Díaz-Muñoz }^{(52)} \\
2005\end{array}$ & $\begin{array}{l}11 \text { enfermeras. } \\
4 \text { aux. enferm. }\end{array}$ & $14^{\prime} 86 \pm 7^{\prime} 43$ & $6 ’ 26 \pm 4 ’ 69$ & $35^{\prime} 06 \pm 7^{\prime} 51$ \\
\hline $\begin{array}{l}\text { Casado }^{(56)} \\
2005\end{array}$ & 29 enfermeras. & $17^{\prime} 2 \pm 3^{\prime} 5$ & $8^{\prime} 1 \pm 2$ & $21^{\prime} 4 \pm 3{ }^{\prime} 9$ \\
\hline Muñoz ${ }^{(51)}$ & 10 enfermeras & $33^{\prime} 5$ & $11 ’ 3$ & $30 ’ 9$ \\
\hline 2002 & $\begin{array}{c}\text { que salieron de } \\
\text { UCI. }\end{array}$ & $\begin{array}{c}50 \% \text { niveles } \\
\text { elevados. }\end{array}$ & $\begin{array}{c}50 \% \text { niveles } \\
\text { elevados. }\end{array}$ & $\begin{array}{c}40 \% \text { niveles } \\
\text { bajos. }\end{array}$ \\
\hline
\end{tabular}

El agotamiento emocional es alto si la puntuación es $>29$, medio entre 18 y 29 y bajo si es $<18$. La despersonalización es máxima cuando la puntuación es $>11$, media si se encuentra entre 6 y 11 y baja si es $<6$ puntos.

Realización personal: a más puntuación, mayor sensación de realización personal en el trabajo; es alta si la puntuación es $>39$ puntos, media entre 34 y 39 y baja si es $<34$.

Al estudiar las tres dimensiones del burnout por separado, cinco estudios midieron niveles altos $^{(24,53,54)}$ y medios ${ }^{(50,57)}$ de cansancio emocional, atribuyéndolo a diferentes causas: García $^{(24)}$, Ríos ${ }^{(53)}$ y Frade ${ }^{(54)}$ encontraron mayor cansancio emocional en los profesionales de más antigüedad y edad, según García ${ }^{(24)}$ porque querían abandonar el servicio y no habían podido. Carmona ${ }^{(50)}$ encontró que las enfermeras contratadas tenían niveles más altos de cansancio emocional que las fijas debido a la incertidumbre e inseguridad laboral que les provocaba su situación, resultado opuesto al obtenido por Solano ${ }^{(49)}$, quien midió niveles más bajos de cansancio emocional en los contratados porque "desconectaban" al ser cesados.

En cuanto a la despersonalización, García ${ }^{(24)}$ y Santana ${ }^{(57)}$ obtuvieron niveles altos (García ${ }^{(24)}$ lo atribuyó a la presión asistencial a que estaba sometida su muestra), mientras $\operatorname{Rios}^{(53)}$ y Díaz-Muñoz ${ }^{(52)}$ encontraron niveles medios. Solano ${ }^{(49)}$ midió niveles más altos de despersonalización en el turno de noche y lo atribuyó a la menor comunicación y administración de cuidados a los pacientes en ese turno. 
La realización personal era baja en los estudios de $\operatorname{García}^{(24)}$, Santana ${ }^{(57)}$ y Ríos ${ }^{(53)}$, asociándose significativamente a trabajar en el turno de noche ${ }^{(24)}$ posiblemente por la escasa integración y participación activa con el resto del equipo y las características propias del turno.

Los artículos comparando el burnout en UCI y plantas de hospitalización encontraron los mismos niveles en ambos grupos de profesionales ${ }^{(52,57)}$. A su vez, Ríos ${ }^{(58)}$ comparó el burnout de las enfermeras de urgencias y $\mathrm{UCI}$, encontrando mayor agotamiento emocional en las que trabajaban en $\mathrm{UCI}$, resultado opuesto al obtenido por Casado ${ }^{(56)}$.

Muñoz ${ }^{(51)}$ comparó el nivel de burnout de las enfermeras que habían salido de UCI tras cinco o más años de actividad con las que continuaban en la misma: el 50\% de las enfermeras que se habían trasladado tenían niveles altos de cansancio emocional y despersonalización y el $40 \%$ niveles bajos de realización personal y posiblemente habían salido de UCI por "estar quemadas", mientras sólo el 10\% de la que seguían en UCI presentaba estos niveles.

\section{Familiares de los pacientes}

Velasco(90), en 2005, determinó que en 93 de 98 UCl estudiadas existían limitaciones respecto al número de visitas (el $64 \%$ permitía 2 visitas al día), duración (media hora en el $59 \%$ ), familiares por visita ( 2 en el $52 \%$ ) y familiares de forma simultánea ( 2 en el $65 \%$ de unidades).

Varios autores analizaron la opinión de las enfermeras sobre la política de visitas seguida en sus unidades con resultados opuestos; Zaforteza ${ }^{(59)}$, en un estudio cualitativo con 8 enfermeras de $3 \mathrm{UCl}$, encontró que muchas opinaban que la duración de la visita era suficiente y el momento de la misma podía ser una fuente de angustia para el familiar, por lo que no debía aumentarse su duración y la enfermera no debía realizar ningún tipo de actividad con el paciente (salvo que fuera necesario) para no alarmar al familiar; también el $70 \%$ de los profesionales del trabajo de Santana ${ }^{(60)}$ consideraron adecuado el tiempo de visita y, el $84 \%$, el número de familiares que entraba en la unidad. Por el contrario, en el estudio de Burgos ${ }^{(61)}$, el $71 \%$ de las enfermeras creía que eran insuficientes el horario y duración de la visita, aunque el $85 \%$ se mostró partidario de poder restringirlos en función de las características del paciente y su familia.

Dos unidades publicaron sus experiencias tras modificar sus normas de visita. Marco ${ }^{(62)}$ recabó la opinión de las enfermeras tras ampliar las visitas que, de ser restrictivas en horario, duración y ver al paciente a través de una ventana, habían pasado a ser abiertas, permanecer los familiares periodos de tiempo con el paciente y flexibilizar el horario según las necesidades del enfermo y familia; el 93\% de las enfermeras se mostraron satisfechas con las nuevas normas y todas opinaron que la familia proporcionaba apoyo emocional al paciente, minimizaba su aburrimiento y, el 91\%, que incrementaba su deseo de vivir; asimismo, el $100 \%$ creía que aumentaba la satisfacción de la familia, el 93\% que disminuía la ansiedad de ésta, le proporcionaba un mayor apoyo del equipo de enfermería y mayor seguridad de que el paciente recibía los mejores cuidados.

En la UCI del Hospital de Valme dieron un paso más al implicar al familiar en los cuidados básicos del enfermo (alimentación, higiene general y de la boca, movilización cama/sillón y pasiva de articulaciones) y Rodríguez ${ }^{(63)}$, en 2003, publicó los resultados obtenidos al evaluar los cambios; el $91 \%$ de las enfermeras afirmó que la participación en los cuidados proporcionaba apoyo emocional al paciente, el $82 \%$ que disminuía su aburrimiento y el $67 \%$, que incrementaba su deseo de vivir; asimismo, el 97\% del personal de enfermería opinó que 
aumentaba la satisfacción de la familia, el $85 \%$ que disminuía su ansiedad y el $73 \%$ que la familia recibía mayor información y le facilitaba un conocimiento más real de la situación del enfermo. Como contrapartida, el $85 \%$ en el estudio de Marco ${ }^{(62)}$ y el $61 \%$ en el de Rodríguez $^{(63)}$ creían que la presencia de la familia interrumpía o posponía los cuidados de enfermería, especialmente los procedimientos dolorosos, y producía una mayor carga física y psíquica al personal ${ }^{(62,63)}$.

Otro aspecto investigado ha sido la relación de las enfermeras con los familiares; éstas, en el estudio de Zaforteza ${ }^{(59)}$, consideraban a los familiares elementos externos a la unidad y al proceso de cuidar al paciente crítico y decidían relacionarse más o menos con ellos según su grado de afinidad, que podía estar relacionada con aspectos emocionales, de nivel educacional o de otra naturaleza; en el trabajo de Díaz ${ }^{(64)}$, las enfermeras no asumían como sujeto de cuidados a los familiares del paciente crítico y, el $66 \%$ en el estudio de Santana ${ }^{(60)}$, creía que su relación con los familiares era poco fluida o inexistente.

Respecto a la información a los familiares, Zaforteza ${ }^{(65)}$ encontró que las enfermeras percibían al médico como el dueño del proceso de información al paciente y familiares, consideraban que su papel como informadoras era insuficiente y deseaban participar más en el proceso de atención a los familiares para establecer con ellos una relación terapéutica; en los estudios de Díaz ${ }^{(64)}$ y Santana ${ }^{(60)}$ las enfermeras opinaban que se utilizaba jerga médica para informar a los familiares y estos, en ocasiones, no entendían lo que se les decía pero no pedían explicaciones. También el entorno físico de la UCI (salas de espera e información) ha sido calificado como incómodo ${ }^{(60,64)}$ o falto de la intimidad necesaria para que los familiares manifestasen abiertamente sus necesidades psicológicas y afectivas ${ }^{(60,61,64)}$.

Pérez ${ }^{(66)}$ encontró que las preguntas más frecuentes realizadas por la familia de los pacientes ingresados al personal de enfermería eran referentes a constantes vitales, monitorización y aparataje, horarios de visita y cuidados. El 54\% de las enfermeras creía que debía facilitar información sobre los cuidados de enfermería, un $20 \%$ que también debería informar sobre la evolución y el estado del paciente y un $5 \%$ que no debía dar ningún tipo de información, resultado similar al obtenido por Santana ${ }^{(67)}$, donde el $47 \%$ de las enfermeras sólo hablaba en algunas ocasiones con el familiar sobre la enfermedad o el tratamiento aplicado.

Finalmente, varios autores ${ }^{(59,60,64,67)}$ afirmaban que era necesario establecer estándares de calidad para la atención a las familias e integrarlas en el plan de cuidados de enfermería, para lo que era necesario formar a los profesionales en habilidades psico-sociales y elaborar protocolos de atención a los familiares.

\section{Muerte en UCI}

Aunque al $80 \%$ de las enfermeras ${ }^{(25)}$ la muerte de un paciente no les provocaba sentimientos de culpabilidad, la mitad de las mismas en el trabajo de Diez ${ }^{(18)}$ decía sentir mucho o bastante el fallecimiento de un paciente en concreto, calificándolo en otro estudio incluso como una pérdida ${ }^{(35)}$. El $55 \%$ de las profesionales en el trabajo de Marín ${ }^{(68)}$ evitaba hablar de la muerte $y$, junto con la retirada de sondas, tubos... al cadáver, la muerte era lo que más había impactado a las enfermeras en sus comienzos en $\mathrm{UCI}^{(29)}$. Asimismo, el contacto con la misma había afianzado el sistema de valores del $80 \%$ de las participantes en el trabajo de Notivol $^{(69)}$, la mitad de las cuales manifestó que su trabajo asistencial no finalizaba al fallecer el paciente. 
González ${ }^{(70)}$, en un estudio en la UCl pediátrica del Hospital Gregorio Marañón, determinó que las enfermeras elaboraban estrategias de forma inconsciente redefiniendo el concepto de muerte en "buena" o "mala", evitaban crear lazos sentimentales que reagudizasen el sentimiento de pérdida en caso de fallecimiento del niño, aludían a los cuidados post-mortem y a la muerte misma con metáforas y evitaban símbolos o elementos cargados de fuertes connotaciones respecto a ésta.

Con relativa frecuencia, los profesionales de $\mathrm{UCl}$ cuidan a pacientes que van a morir y a sus familiares; según las enfermeras de $5 \mathrm{UCl}$ de la Comunidad de Navarra, lo que más ayudaba en esta tarea era que todos los médicos estuvieran de acuerdo con el enfoque de los cuidados, la familia aceptase el hecho y dispusiera de un entorno pacífico y digno tras el exitus y, lo que menos ayudaba, era tener un miembro del comité de ética en los pases de visita diarios. El máximo obstáculo correspondía a que el paciente tuviera dolor difícil de controlar, proporcionar cuidados dolorosos al paciente en estado terminal y que los médicos tuvieran una actitud evasiva y evitasen a la familia ${ }^{(71)}$.

El $72 \%$ de las enfermeras en el trabajo de Santana ${ }^{(72)}$ tenían la impresión de que, a los pacientes que iban a morir, se les mantenía vivos durante demasiado tiempo y, el 53\%, que los deseos del paciente no se tenían en cuenta; las mismas puntuaron los cuidados que ofrecían con 8,66 $\pm 0,99$ sobre $10^{(72)}$.

Aunque Notivol ${ }^{(69)}$, Marín ${ }^{(68)}$, Gonzalez ${ }^{(70)}$ y Del Barrio ${ }^{(71)}$ concluían que era necesaria preparación específica para enfrentarse a la muerte y dar apoyo al paciente y su familia, sólo el $28 \%$ de las enfermeras ${ }^{(72)}$ había realizado algún curso acerca del proceso del morir para enfermería, habiendo aprendido el $44 \%{ }^{(69)}$ de la experiencia.

\section{Ética en UCI}

Camaño ${ }^{(73)}$, en 1988, llevó a cabo un estudio con 86 enfermeras de UCl de 4 hospitales, en el cual el $69 \%$ consideraba importante que el enfermo estuviera informado del proceso patológico que sufría, el 57\% que conociera el pronóstico de su enfermedad y los riesgos de la aplicación de ciertos fármacos, técnicas, etc., siendo el mismo porcentaje el que afirmaba que no se facilitaba esta información a los pacientes. En 1994, en una investigación en la UCl de 28 hospitales, Abizanda ${ }^{(74)}$ encontró que el $94 \%$ de las enfermeras opinaban que se debía informar al paciente y familia de su situación y posibilidades y, el $98 \%$, que la familia debía recibir la misma información que el paciente, sobre todo si éste no era competente; el $74 \%$ creía que, en casos de conflicto de opinión, el médico podía imponer su mejor juicio, aunque el $93 \%$ se mostró a favor de solicitar consentimiento informado al enfermo cuando fuera posible. En este mismo estudio ${ }^{(74)}$, el $98 \%$ de las enfermeras se declaró partidaria de las órdenes de no resucitación en determinados supuestos y el $92 \%$ de establecer límites terapéuticos bajo determinadas condiciones y tomar las decisiones considerando la calidad de vida posterior. Además, el $87 \%$ creían necesario o conveniente la existencia de comités de bioética.

En 2001, Gómez ${ }^{(75)}$ analizó la respuesta de las enfermeras ante 5 casos clínicos representativos de conflictos éticos, en los que debían decidir si instaurar o no ventilación mecánica; en 4 de los supuestos la respuesta de éstas coincidía con el comité de ética del hospital pero las decisiones de limitación del esfuerzo terapéutico no eran mayoritariamente aceptadas, incluso en escenarios que entraban en el terreno de la futilidad.

Otro aspecto estudiado ha sido la intimidad de los enfermos. Según Arévalo ${ }^{(76)}$, ésta era una necesidad sentida por los profesionales de enfermería, quienes opinaban que el cuidado 
enfermero debía garantizar la intimidad y privacidad de los pacientes y el cuidador debía ser consciente de la intimidad que se le brindaba y que debía ser tratada con sensibilidad y respeto; Camaño ${ }^{(73)}$ encontró que el $60 \%$ ponía los medios para garantizar la comodidad del paciente en la higiene y eliminacion y el $71 \%$ no daba publicidad a las confidencias de los enfermos sobre su vida privada, resultados coincidentes con el estudio de Arévalo ${ }^{(76)}$.

Finalmente, la humanización de la asistencia, según las enfermeras en el estudio de Rubio $^{(27)}$, requiere tiempo, recursos, buena relación intergrupal y el compromiso explícito de la institución.

\section{Evaluación de conocimientos sobre técnicas y/o cuidados.}

Bien con el objetivo de identificar posibles carencias y establecer programas de formación específicos, bien en el seno de programas de mejora de la calidad, varios autores han evaluado los conocimientos de las enfermeras sobre diferentes técnicas y cuidados que conforman el día a día de una $\mathrm{UCI}^{(77-85)}$. Los objetivos y principales conclusiones de estos estudios se pueden consultar en la tabla V.

Tabla V.- Estudios de evaluación de conocimientos sobre técnicas y/o cuidados.

\begin{tabular}{|c|c|c|c|}
\hline $\begin{array}{l}\text { Autor y } \\
\text { año del } \\
\text { estudio. }\end{array}$ & $\begin{array}{l}\text { Particip } \\
\text { antes. }\end{array}$ & Objetivos. & Resultados/Conclusiones. \\
\hline $\begin{array}{c}\underset{(77)}{\text { Navarro }} \\
2001\end{array}$ & $\begin{array}{c}42 \text { enf. } \\
12 \\
\text { aux.enf. }\end{array}$ & $\begin{array}{l}\text { - Conocer los factores que alteraban el } \\
\text { descanso/sueño de los pacientes. } \\
\text { - Conocer si en los registros de } \\
\text { enfermería constaba cómo había } \\
\text { dormido el paciente. }\end{array}$ & $\begin{array}{l}\text { - A los pacientes les dificultaba dormir la } \\
\text { actividad del box y realizarles técnicas. } \\
\text { - Enfermería detectaba bien cómo habían } \\
\text { dormido los pacientes pero no tenía el hábito } \\
\text { de registrarlo. }\end{array}$ \\
\hline $\begin{array}{c}\text { Molano } \\
2003\end{array}$ & 131 enf. & $\begin{array}{l}\text { - Identificar los diferentes modelos de } \\
\text { manejo de técnicas continuas de } \\
\text { reemplazo renal en la Comunidad de } \\
\text { Madrid. } \\
\text { - Conocer el papel que asume } \\
\text { enfermería en cada uno de ellos. } \\
\text { - Evaluar el nivel de conocimientos de } \\
\text { estas terapias y detectar los principales } \\
\text { problemas percibidos durante su } \\
\text { manejo. }\end{array}$ & $\begin{array}{l}\text { - El modelo más frecuente es aquel en que la } \\
\text { enfermera monta y controla la terapia y el } \\
\text { intensivista pauta flujos, extracción y } \\
\text { anticoagulación. } \\
\text { - La enfermería de UCC puede y debe } \\
\text { manejar las TCRR adecuándose la ratio } \\
\text { enfermera/paciente y estableciendo } \\
\text { programas de formación. } \\
\text { - El principal problema es el déficit de } \\
\text { conocimientos para resolver complicaciones. }\end{array}$ \\
\hline$\underset{(79)}{\text { Navarro }}$ & 51 enf. & $\begin{array}{l}\text { - Analizar la satisfacción de los } \\
\text { profesionales con el informe de alta de } \\
\text { enfermería. } \\
\text { - Determinar las modificaciones que } \\
\text { proponían como paso previo al diseño } \\
\text { de un nuevo informe. }\end{array}$ & $\begin{array}{l}\text { - El 97\% consideró el informe útil para las } \\
\text { enfermeras de planta, necesario en la } \\
\text { documentación y una buena forma de } \\
\text { comunicación entre servicios. } \\
\text { - Los profesionales proponían una serie de } \\
\text { modificaciones. }\end{array}$ \\
\hline
\end{tabular}




\begin{tabular}{|c|c|c|c|}
\hline $\begin{array}{l}\text { Ania }^{(80)} \\
2004 .\end{array}$ & 34 enf. & $\begin{array}{l}\text { - Evaluar la competencia práctica de } \\
\text { las enfermeras y sus conocimientos } \\
\text { científicos sobre la aspiración } \\
\text { endotraqueal de secreciones. } \\
\text { - Analizar si existen discrepancias } \\
\text { entre la competencia práctica y los } \\
\text { conocimientos científicos. }\end{array}$ & $\begin{array}{l}\text { - Las enfermeras tenían unos conocimientos } \\
\text { científicos del procedimiento mejores que su } \\
\text { competencia práctica. } \\
\text { - Se encontraron discrepancias entre la } \\
\text { práctica y los conocimientos en varios } \\
\text { aspectos evaluados. }\end{array}$ \\
\hline $\begin{array}{c}\text { Montial }^{(81} \\
2005\end{array}$ & $\begin{array}{l}30 \text { enf. } \\
13 \\
\text { aux.enf. }\end{array}$ & $\begin{array}{l}\text { - Determinar la destreza y precisión } \\
\text { con la que el personal de enfermería } \\
\text { elevaba la cabecera de } \\
\text { la cama de los pacientes a } 30 \text { y a } 45^{\circ} \text {. }\end{array}$ & $\begin{array}{l}\text { - Los profesionales situaban la cabecera de la } \\
\text { cama por debajo de la inclinación } \\
\text { recomendada. } \\
\text { - Es necesaria la existencia de un medidor de } \\
\text { inclinación en las camas. }\end{array}$ \\
\hline $\begin{array}{l}\underset{(82)}{\text { Gutierrez }} \\
2005\end{array}$ & $\begin{array}{l}8 \text { enf. } \\
6 \\
\text { aux.enf. }\end{array}$ & $\begin{array}{l}\text { - Conocer los errores al calcular los } \\
\text { balances hídricos de los pacientes. } \\
\text { - Proponer métodos para reducirlos sin } \\
\text { incrementar las cargas de trabajo del } \\
\text { personal. }\end{array}$ & $\begin{array}{l}\text { - Existía poca concordancia con los valores } \\
\text { reales en las botellas de diuresis. } \\
\text { - La valoración subjetiva del volumen } \\
\text { contenido en cuñas, pañales, empapadores, } \\
\text { compresas y gasas no era un método } \\
\text { apropiado por su escasa fiabilidad. }\end{array}$ \\
\hline$\underset{(83)}{\text { Navarro }}$ & 42 enf. & $\begin{array}{l}\text { - Establecer el grado de satisfacción } \\
\text { con un nuevo informe de alta de } \\
\text { enfermería. }\end{array}$ & $\begin{array}{l}\text { - El } 86 \% \text { de las enfermeras calificó el nuevo } \\
\text { informe como mejor o mucho mejor que el } \\
\text { anterior. }\end{array}$ \\
\hline $\begin{array}{l}\text { Rodrígue } \\
\mathrm{z}^{(84)} \\
2007\end{array}$ & 40 enf. & $\begin{array}{l}\text { - Conocer la formación, } \\
\text { concienciación e implicación del } \\
\text { personal respecto a la valoración del } \\
\text { dolor. }\end{array}$ & $\begin{array}{l}\text { - Un } 75 \% \text { conocía las escalas y herramientas } \\
\text { para la valoración del dolor. } \\
\text { - Para las enfermeras con } 4 \text { o más años de } \\
\text { experiencia en UCI, el control del dolor era } \\
\text { lo más relevante y le otorgaban una } \\
\text { puntuación de } 9 \text { sobre } 10 . \\
\text { - El personal con menos de } 4 \text { años de } \\
\text { experiencia daba al dolor una puntuación de } \\
7 \text { sobre } 10 .\end{array}$ \\
\hline$\underset{(85)}{\text { Quesada }}$ & 120 enf. & $\begin{array}{l}\text { - Determinar el grado de conocimiento } \\
\text { de las enfermeras sobre las } \\
\text { recomendaciones basadas en } \\
\text { evidencias de prevención y } \\
\text { tratamiento de UPP. }\end{array}$ & $\begin{array}{l}\text { El grado de conocimiento sobre las } \\
\text { recomendaciones de prevención de úlceras } \\
\text { por presión distaba mucho de ser el ideal. } \\
\text { - De } 22 \text { recomendaciones basadas en } \\
\text { evidencias ( } 11 \text { intervenciones preventivas y } \\
11 \text { de tratamiento), el } 100 \% \text { sólo conocía una } \\
\text { media de } 12 \text {. } \\
\text { - Se conocían mejor las recomendaciones de } \\
\text { prevención que las de tratamiento. }\end{array}$ \\
\hline
\end{tabular}

\section{Temas informáticos en UCl.}

En los últimos años se han puesto en funcionamiento diversas aplicaciones informáticas en las unidades de cuidados intensivos, aplicaciones evaluadas en diversos trabajos. En el estudio de González ${ }^{(86)}$, en cuya unidad de críticos se implantó el sistema CareVue ${ }^{\circledR}$, el $52 \%$ de las enfermeras lo consideraba un método de registro más claro al dejar constancia del trabajo que realizaba la enfermería y el $60 \%$ afirmó que disponía de más tiempo para la asistencia al paciente, por lo que concluía que el procedimiento informatizado suponía una mejora del método de trabajo frente al tradicional en bolígrafo y papel. 
Por contra, García ${ }^{(87)}$ concluyó que el aplicativo Gacela ${ }^{\circledR}$, puesto en marcha en su unidad en 2003, era una herramienta inadecuada para el trabajo en UCI al considerar el $87 \%$ de los profesionales que los registros de este sistema eran peores o mucho peores que los de la grafica estándar y puntuar su facilidad de uso y utilidad con un 3 sobre 10.

En la UCI del Hospital General Universitario de Alicante diseñaron una aplicación informática propia para la cumplimentación y archivo de los informes de alta de enfermería ${ }^{(88)}$; al evaluarla, el $93 \%$ de las enfermeras la utilizaba de forma habitual y sólo el $32 \%$ volvería a elaborar los informes en papel.

Respecto al uso que las enfermeras hacían de la red internet, un trabajo con 200 enfermeras $^{(89)}$ determinó que, el $94 \%$, enviaba/recibía correo electrónico, el $89 \%$ buscaba información sobre salud, el $78 \%$ recababa información sobre otros temas y el $70 \%$ consultaba revistas científicas sanitarias.

\section{DISCUSIÓN}

Los trabajos de investigación publicados en España con las enfermeras de UCI como sujeto a estudio se han incrementando en los últimos años (especialmente a partir del año 2000), sus autoras son, mayoritariamente $(71 \%)$, enfermeras y supervisoras que trabajan en unidades de cuidados intensivos e investigan en colaboración (casi la mitad de los estudios están firmados por 3 ó 4 autoras), resultados que coinciden con los obtenidos en los estudios sobre producción científica en enfermería de cuidados intensivos ${ }^{(4,6-8,91)}$, posiblemente debido a que los artículos estudiados son un subconjunto de los analizados por estos autores.

Los trabajos que describen a los profesionales de $\mathrm{UCl}$ y sus condiciones de trabajo son muy antiguos (los más recientes datan de 1999), por lo que sería conveniente realizar un estudio actualizado a nivel nacional, englobando al mayor número posible de unidades, que aportaría conocimiento sobre diversos aspectos de las enfermeras de cuidados intensivos (de las que, incluso, desconocemos su número) como datos sociodemográficos, condiciones laborales tras la aparición de nuevos elementos como las unidades de semicríticos, nuevos modelos de gestión de hospitales y posibilitaría planificar necesidades futuras de profesionales (puesto que una enfermera precisa entre 12 y 36 meses para adquirir soltura y seguridad en el trabajo en $\mathrm{UCl}^{(17,19)}$ ), establecer comparaciones con la realidad de otros países de nuestro entorno europeo y mostrar tanto a la sociedad como al resto de profesionales sanitarios el papel clave de estas enfermeras, trabajo no siempre bien conocido ${ }^{(92,93)}$.

Los temas en los que se centran la mayoría de los estudios incluidos en este análisis (clima laboral, burnout, formación de los profesionales) coinciden con las prioridades de investigación para la enfermería de UCl en trabajos internacionales ${ }^{(94,95)}$; en nuestro país, donde la enfermería de cuidados intensivos y coronarios se encuentra en pleno desarrollo, con un crecimiento rápido en el número de artículos ${ }^{(6)}$, sólo un estudio en 1994 abordó las prioridades de investigación para estos profesionales ${ }^{(96)}$, situando la relación entre tecnología y humanización y la formación en los primeros lugares.

Un asunto no resuelto es el de las plantillas de UCl; aunque unos autores ${ }^{(97,98)}$ han propuesto que su cálculo se base en la complejidad de la unidad y las cargas de trabajo de los enfermos, otros autores opinan que sería adecuado legislar una ratio enfermera/paciente ${ }^{(99)}$ para adecuarlas a la realidad europea; en cualquier caso, diversos 
trabajos demuestran que a mayor número de enfermeras, mejores resultados de cuidados $^{(100,101)}$.

Los estudios centrados en los familiares de los pacientes, que comienzan en el año 2000, traducen la tendencia creciente a la flexibilización en la política de visitas de las UCI, el cambio en la apreciación de los familiares (que pasan a ser objetivo de los cuidados de enfermería) y su implicación en los cuidados del paciente, línea de investigación que continúa avanzando ${ }^{(102-104)}$.

Los trabajos sobre aspectos éticos, como la intimidad del paciente o la muerte del mismo en $\mathrm{UCI}$, que han sido objeto de menor número de estudios, posiblemente vayan aumentando debido al aumento de la esperanza de vida de la población y las posibilidades de la tecnología en soporte vital.

\section{CONCLUSIONES}

- La mayor parte de los estudios sobre las enfermeras que trabajan en UCI estaban escritos por las propias enfermeras que trabajaban en UCI.

- Los estudios que ofrecían un perfil de las enfermeras eran antiguos y sería necesaria su actualización.

- Las enfermeras calificaban su trabajo como estresante debido al estado de los pacientes y las condiciones de trabajo, si bien en la mayoría de unidades estudiadas, los niveles de burnout eran medios o bajos y equiparables a los de las unidades de hospitalización.

- Las enfermeras solicitaban formación en temas éticos, de apoyo al paciente y cuidados a la familia, ante la muerte y en determinadas técnicas y aspectos del trabajo diario en UCI.

\section{Agradecimientos}

A los autores y colegios profesionales que amablemente me facilitaron los manuscritos para este trabajo.

A Dña. Encarnación Aguilar Cañizares, administrativa de la biblioteca del H.G.U. de Alicante, por su inestimable colaboración y paciencia infinita.

\section{BIBLIOGRAFÍA}

1.- Chover $\mathrm{H}$. Orígenes de las UCls, evolución de la enfermería de Cuidados Intensivos. Primeras reuniones nacionales y creación de la SEEIUC. Enferm Intensiva 1999;10(3):12940.

2.- González Díaz G, García Córdoba F. Modelos organizativos en Medicina Intensiva: el modelo español. En: Gumersindo González Díaz. Libro electrónico de Medicina Intensiva [Monografía en internet]. Disponible: en http://intensivos.uninet.edu/04/0402.html [Consultado 1 junio 2009].

3.- Asiain MC, Marín B. Enfermería intensiva. Actividad científica en la última década (19781987). Enferm Intensiva 1990;dic(4):13-6.

4.- Marín Fernández B, Gallego Caminero G, Martín Sanz E, San Martín Salazar B. Análisis bibliométrico de la producción científica de la enfermería de cuidados intensivos 1988-1992. Enferm Intensiva 1994;dic(4):166-73.

5.- Asiain MC. Investigación de las enfermeras de Cuidados Intensivos en España durante los últimos veinticinco años. Enferm Intensiva 1999;10(3):131-34. 
6.- Lizarbe Chocarro M. La Enfermería de cuidados intensivos y coronarios en España: análisis bibliométrico. Metas 2007;10(2):9-15.

7.- Lizarbe Chocarro M. Enfermería en Cuidados Intensivos y Coronarios. Análisis bibliométrico de 180 artículos originales. Enferm Intensiva 2007;18(3):126-37.

8. - Lizarbe Chocarro M. Análisis de las referencias bibliográficas de 334 artículos del campo de enfermería de cuidados intensivos y coronarios. Pulso 2008; no 53 (abril):31-33

9.- López Ferrer MT, Osca Lluch J. Análisis de la literatura internacional sobre enfermería en cardiología durante el periodo 1990-1999. Enfermería en Cardiología 2000; 20:22-27.

10.- Bengoechea Ibarrondo MB. Estudios sobre la comunicación paciente enfermera en cuidados intensivos. El estudio de las líneas de investigación predominantes y otras relevantes. Enferm clin 2001;11(6):266-74.

11.- Blanca Gutiérrez JJ, Blanco Alvariño AM, Luque Pérez $M$, Ramírez Pérez MA. Experiencias, percepciones y necesidades en la UCI: revisión sistemática de estudios cualitativos. Enfermeria Global [serie en internet]. 2008 febrero. Disponible en: http://revistas.um.es/eglobal/issue/view/82 [Consultado el 10 de junio de 2009].

12.- Zaforteza C, Sánchez C, Lastra P. Análisis de la literatura sobre los familiares del paciente crítico: es necesario desarrollar investigación en cuidados efectivos. Enferm Intensiva. 2008;19(2):61-70.

13.- Blanca Gutiérrez JJ. La investigación de las enfermeras de cuidados críticos en el campo de las terapias complementarias. Revista páginasenferurg [serie en internet]. 2008 diciembre. Disponible en: http://paginasenferurg.com/revistas/paginasenferurgn00.pdf [Consultado el 1 de febrero de 2009].

14.- Francisco del Rey JC, Chover García MH. Situación de la enfermería en las Unidades de Cuidados Intensivos en España. Rev Rol Enf 1980;27:22-28.

15.- Lozano Quintana MJ, Sánchez-Izquierdo Riera JA, López Castillo MT, Cuenca Solanas M. Enfermería en las unidades de cuidados intensivos. Enferm Intensiva 1994;5(1):9-16.

16.- Cuenca M, Asiaín MC, Marín B. Análisis de la situación de las Unidades de Cuidados Intensivos. Enferm Intensiva 1995;6(3):103-10.

17.- Pascual J, Bueno $P$, Cuenca M, Asiain $M^{a}=$ C, Marín B. Certificación de la competencia profesional de la enfermera de Cuidados Críticos: encuesta de opinión. Enferm Intensiva 1998;9(1):16-20.

18.- Díez Sainz JA, Alegre de Vega C. Aspectos humanos y profesionales del A.T.S. y de U.V.I Tribuna Sanitaria 1986; octubre (32):23-25.

19.- Rodríguez Mondéjar J.J, Navarro Mateu VJ, Burguet Giges AM. Nuestra unidad de cuidados críticos a estudio. Enferm Intensiva 1992;3(3):70-74.

20.- Piriz Campos RM ${ }^{a}$, Sánchez Lozano Y, González Gómez I. Estudio sobre el perfil profesional de las enfermeras/os en unidades especiales. Enferm Intensiva 1999;10(2):5157.

21.- Gámez Perales M, Santano Magariño A, Matías Benayas S, Roca Sánchez S. Estudio del clima laboral en cuatro unidades de críticos de un hospital. Enferm Intensiva 1999;10:120-28.

22.- Ortega Recio $M$, Torres Pérez $M^{\mathrm{a}} \mathrm{P}$. Estudio de la motivación en un servicio de recuperación quirúrgica. Metas 1999;18:6-11.

23.- Navarro-Arnedo JM, Perales-Pastor R, Gómez-Calcerrada-Pérez P, Alba-Muela Mª $M$. Factores relacionados con la larga permanencia laboral de las enfermeras en las unidades de cuidados intensivos. Enferm Clin 2008;18(4):201-04.

24.- García Grau N, Carmona Heredia A, Roca Biosca A, Olona Cabassés M. Síndrome de Burnout en los equipos de Enfermería de Cuidados Intensivos de Cataluña. Metas 2004;7(2):6-12.

25.- Salas Campos L, Pérez Fornós M. Estrés del personal de enfermería en las unidades de cuidados intensivos. Enferm Intensiva 1990;dic., 4: 6-8. 
26.- Esteban de la Torre A, Rivero M, Ayensa A, Gómez-Acebo E. Estudio de la personalidad en un grupo de enfermeras de UCl. Actas Luso Españolas de Neurología, Psiquiatría y Ciencias Afines 1975.Sep-Oct;3(5):327-32.

27.- Rubio Rico L, Cosi Marsans M, Martínez Márquez C, Miró Borrás A, Sans Riba L, Toda Savall $D$ et al. Relaciones interdisciplinarias y humanización en las unidades de cuidados intensivos. Enferm Intensiva 2006;17(4):141-53.

28.- Torné Pérez E, Alaminos Romero R. Ratio enfermera/paciente en cuidados intensivos. Un estudio multicéntrico. Hygia de Enfermería 2003; XV(55):45-50.

29.- Lacasa Esteban L, Poveda Mullet E. Primera Experiencia en UCl. Enfermería Integral 2003;65:21-23.

30.- Navarro Arnedo JM, Orgiler Uranga PE, De Haro Marín S. Guía práctica de enfermería en el paciente crítico. Enferm Intensiva 2005;16(1):15-22.

31.- Palacín Esteban M, Delgado del Val E, Jover Sancho C, Sucorra Sales L, Valls Guallar C, Viera Peña MD. Evaluación de la experiencia enseñanza-aprendizaje del curso de Enfermería Intensiva. Enferm Intensiva 1991;2(1):9-14.

32.- Maull E, Balaguer R, Artiaga M, Bosqué C, Vila J, Solsona JF. La variabilidad en las sistemáticas del trabajo en los servicios de medicina intensiva. Enferm Intensiva 1996;7(3):104-10.

33.- Secorún Torres A, López Riaño JA, Gómez Gamboa E. ¿Afecta el ruido a la persona en las UVI-P? Agora de enfermería 2007;11(2):1115-17.

34.- Ventura Riball MR, Portillo Jáurena E, Verdaguer Cot M, Carrasco Gómez G, Cabré Pericas L, Balaguer Blasco R, et al. Sesiones clínicas conjuntas en UCl y satisfacción de los profesionales. Enferm Intensiva 2002;13(2):68-77.

35.- Rubio Rico L, Aguarón García MํJ, Ferrater Cubells Maㅡ, Toda Salvall D. Vivir la UCl: Diferentes perspectivas. Cul Cuid 2002; VI(12):55-66.

36.- Simón García MํJ, Blesa Malpica AL, Bermejo Pablos C, Calvo Gutierro MaA, Gómez De Enterría Pérez C. Estresores laborales y satisfacción en la enfermería de una unidad de críticos. Enferm Intensiva 2005;16(1):3-14.

37.- Ortega Recio M, Torres Pérez M ${ }^{a} \mathrm{P}$. Estudio de la satisfacción laboral en un servicio de recuperación quirúrgica. Metas 1999;13:19-27.

38.- Ríos Risquez Mำl, Godoy Fernández C. Síndrome de Burnout y satisfacción laboral en profesionales de enfermería de urgencias y cuidados críticos. Tesela [Serie en internet] 2008;3. Disponible en: http://www.index-f.com/tesela/ts3/ts6565.php [Consultado el 21-junio2009].

39.- Esteban A, Gómez-Acebo E, Rivero M, Landin L. Estudio de la personalidad en un grupo de enfermeras de UCl. Med Intensiva 1977;1(2):59-61.

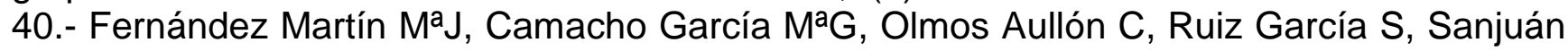
Navais M. Análisis y registro de las agresiones en UCI. Enferm Intensiva 2006;17(2):59-66. 41.- Japón Ruiz D. Sobre una cama de UCl: una experiencia personal. Tempus Vitalis [serie en internet] 2002;2(1):24-27. Disponible en http://www.tempusvitalis.com/descargas/articulos/2002vol2num1/3vivencias21.pdf [Consultado el 1 de septiembre de 2009].

42.- Bellido Vallejo JC. El paso por una unidad de cuidados intensivos y el regreso a la vida. Arch Memoria [serie en internet] 2006; 3(1). Disponible en: http://www.indexf.com/memoria/3/a0613.php [Consultado el 1 de septiembre de 2009].

43.- García León AJ, León Díaz EC, León Díaz I. Estrés en el personal de enfermería de cuidados críticos. Garnata 1999,14:39-67.

44.- Morata Ramírez MA, Ferrer Pérez VA. Interacción entre estrés ocupacional, estrés psicológico y dolor lumbar: un estudio en profesionales sanitarios de traumatología y cuidados intensivos. Mapfre Medicina 2004;15:199-11.

45.- Tomás Sábado J, Fernández Donaire L. Estrés laboral en los profesionales de Enfermería hospitalarios. Metas 2002;45:6-12. 
46.- Fornes J, Gonzalez R, Almendros ML, Crespi M, Gallego G. Respuesta del personal de enfermería intensiva al estrés profesional. Enferm Intensiva 1992;3(1):8-14.

47.- Simarro Blasco JA, Bascuñana Blasco M, Noheda Blasco $M^{a} \mathrm{C}$, Noheda Recuenco $M$, Notario Pacheco B, Guijarro Hernáiz Mạ $J$, Orozco Recuenco Maㅡ. Estrés en la Unidad de Cuidados Intensivos en función de los grados de estilos de aprendizaje. Metas 2009;12(10):62-69.

48.- Surroca Sales L, Badia J, Casanovas M, Herrero I, Oliva Torras E. ¡Me sofoco, busco apoyo en mi compañero!: respuestas del personal de enfermería intensiva ante situaciones estresantes. Enferm Intensiva 1993;4(2):33-39.

49.- Solano Ruiz $M^{a} C$, Hernández Vidal $P$, Vizcaya Moreno $M^{a} F$, Reig Ferrer A. Síndrome de Burnout en profesionales de enfermería de cuidados críticos. Enferm Intensiva 2002;13(1):916.

50.- Carmona Monge FJ, Sanz Rodríguez LJ, Marín Morales D. Relaciones entre el Síndrome de Burnout en profesionales de enfermería. Factores sociodemográficos y reactividad al estrés. Enfermería Científica 2002;ene-feb.238-239:33-39.

51.- Muñoz París $M^{a}-J$, Pérez Ruiz IMaㅡ, Granero Molina J. Inmunidad de Enfermería a los efectos del Burnout. Metas 2002;51:6-9.

52.- Díaz-Muñoz Crespo MậJ. Síndrome del quemado en profesionales de Enfermería que trabajan en un hospital monográfico para pacientes cardíacos. Nure Investigación [serie en internet] septiembre/octubre $2005 . \quad$ Disponible en: http://www.nureinvestigacion.es/FICHEROS ADMINISTRADOR/ORIGINAL/Original18.pdf

[Consultado el 1 de febrero de 2009].

53.- Ríos Risquez MI, Peñalver Hernández F, Godoy Fernández C.Burnout y salud percibida en profesionales de enfermería de Cuidados Intensivos. Enferm Intensiva 2008;19(4):16978.

54.- Frade Mera MaJ, Vinagre Gaspar R, Zaragoza García I, Viñas Sánchez S, Antúnez Melero E, Álvarez González S. Síndrome de burnout en distintas Unidades de Cuidados Intensivos. Enferm Intensiva 2009;20(4):131-40.

55.- Carmona Monge FJ, Sanz Rodríguez LJ, Marín Morales D. Clima social laboral y síndrome de burnout en profesionales de enfermería. Enfermería Científica 2002;244245;82-87.

56.- Casado Moragón A, Muñoz Durán G, Ortega González A. Burnout en profesionales de los servicios paliativos, intensivos y urgencias de un gran hospital. Med Clin (Barc) 2005;124:554-5.

57.- Santana Cabrera L, Hernández Medina E, Eugenio Robaina P, Sánchez-Palacios M, Pérez Sánchez R, Falcón Moreno R. Síndrome de burnout entre el personal de enfermería y auxiliar de una unidad de cuidados intensivos y el de las plantas de hospitalización. Enferm Clin 2009;19(1):31-34.

58.- Ríos Risquez Mํal, Godoy Fernández C, Peñalver Hernández F, Alonso Tovar A, López Alcaraz F, López Romera A et al. Estudio comparativo del burnout en personal de enfermería de cuidados intensivos y urgencias. Enferm Intensiva 2008;19(1):2-13.

59.- Zaforteza Lallemand C, de Pedro Gómez JE, Gastaldo D, Lastra Cubel P, SánchezCuenca López P. ¿Qué perspectiva tienen las enfermeras de unidades de cuidados intensivos de su relación con los familiares del paciente crítico? Enferm Intensiva 2003;14(3):109-19.

60.- Santana Cabrera L, Sánchez Palacios M, Hernández Medina E, García Martul M, Eugenio Robaina P, Villanueva Ortiz A. Necesidades de los familiares de pacientes de Cuidados Intensivos: percepción de los familiares y del profesional. Med Intensiva 2007;31(6):273-80.

61.- Burgos Naranjo RMª , Cano Peregrina MaA, García Córdoba C, Lage López A, López Pelegrí M. Visitas de familiares a pacientes ingresados en la Unidad de Cuidados Coronarios: Opinión de todos los implicados. Enfermería en Cardiología [serie en internet] 
2000;20:30-41. Disponible en: http://www.enfermeriaencardiologia.com/revista/res2003.htm [consultado 2 de julio de 2008].

62.- Marco Landa L, Bermejillo Eguía I, Garayalde Fernández de Pinedo N, Sarrate Adot I, Margall Coscojuela MA, Asiain Erro MC. Creencias y actitudes de las enfermeras de cuidados intensivos sobre el efecto que la visita abierta produce en el paciente, familia y enfermeras. Enferm Intensiva 2000;11(3):107-17.

63.- Rodríguez Martínez $M^{a} \mathrm{C}$, Rodríguez Morilla F, Roncero Del Pino A, Morgado Almenara Mıㅣ, Bannik JT, Flores Caballero LJ et al. Implicación familiar en los cuidados del paciente crítico. Enferm Intensiva 2003;14(3):96-108.

64.- Díaz Sánchez, Nuria Díaz Sánchez. Ampliando horizontes: Una experiencia sobre las vivencias de los familiares de pacientes críticos. Nure investigación [serie en internet] noviembre 2004.2 Disponible en: http://www.nureinvestigacion.es/originales detalle.cfm?ID ORIGINAL=76\&ID ORIGINAL IN I=1 [Consultado el 2 de marzo de 2009].

65.- Zaforteza C, Gastaldo D, Sánchez-Cuenca P, de Pedro JE, Lastra P. Relación entre enfermeras de Unidades de Cuidados Intensivos y familiares: Indicios para el cambio. Nure Investigación [serie en internet] Marzo 2004. Disponible en: http://www. nureinvestigacion.es/originales obj.cfm?id original=59\&ID ORIGINAL INI=1

[Consultado el 1 de marzo de 2009].

66.- Pérez Fernández $M^{a} C$, Najarro Infante FR, Dulce García MA, Gallardo Jiménez N, Fernández Fernández A. Comunicación: Una necesidad para el Paciente-Familia. Una competencia de Enfermería. Páginasenferurg.com [serie en internet] Septiembre 2009. Disponible en: http://paginasenferurg.com/revistas/paginasenferurgn03.pdf [Consultado el 1 de marzo de 2010].

67.- Santana Cabrera L, Yañez Quintana B, Martín Alonso J, Ramírez Montesdeoca F, Roger Marrero I, Susilla Pérez de la Blanca A. Actividades de comunicación del personal de enfermería con los familiares de pacientes ingresados en una unidad de cuidados intensivos. Enferm Clin 2009;19(6):335-39.

68.- Marín Fernández B, Los Arcos Moreno Y, Arriazu Al, Medrano R, Aragón MãL. La muerte en $\mathrm{UCl}$. ¿Cómo reaccionan los profesionales de enfermería?. Enfermería Científica 1993;140:26-31.

69.- Notivol Tejero MP, Santos Laraña MA, Gabari Gambarte MI, Pollán Rufo M. Ayudar a morir en UCl: percepción de las necesidades de los profesionales. Enferm Clin 1993;3(2):43-47.

70.- González Gil T. Estrategias culturales para el afrontamiento de la muerte infantil en una Unidad de Cuidados Intensivos Pediátricos. Enferm Intensiva 2008;19(3):113-22.

71.- Barrio Linares M, Jimeno San Martín L, López Alfaro P, Ezenarro Muruamendiaraz A, Margall Coscojuela MA, Asiain Erro $\mathrm{M}^{\mathrm{a}} \mathrm{C}$. Cuidados del paciente al final de la vida: ayudas y obstáculosque perciben las enfermeras de Cuidados Intensivos. Enferm Intensiva 2007;18(1):3-14.

72.- Santana Cabrera L, Sánchez-Palacios M, Rodríguez Guedes C, Hernández Medina E. Calidad del morir y de la muerte en una unidad de cuidados intensivos: percepción de la enfermería. Enferm Clin 2008;18(3):172.

73.- Camaño Puig A, Benavent Garcés A, Cuesta Zambrana A. Aspectos éticos en UCl. Rev Rol Enf 1988;114:33-37.

74.- Abizanda R, Almendros Corral L, Balerdi Pérez B. Aspectos éticos de la medicina intensiva. Resultados de una encuesta de opinión. Med Clin 1994;102(14):521-526.

75.- Gómez Rubí JA, Gómez Company JA, Sanmartín Monzó JL, Martínez Fresneda M.Conflictos éticos en la instauración de la ventilación mecánica: análisis de la actitud de profesionales de Cuidados Críticos, Emergencia y estudiantes. Rev Clín Esp 2001;201:37177. 
76.- Arévalo Rubert MํJ, Maqueda Palau M, Pérez Juan E, Amorós Cerdá SM. La intimidad en la Unidad de Cuidados Intensivos: Perspectiva enfermera categorizada desde los sentidos. Etica de los Cuidados [Serie en internet] enero-junio 2009. Disponible en: http://www.index-f.com/eticuidado/n3/et6827.php [Consultado el 13 de Noviembre de 2009]. 77.- Navarro Arnedo JM, de Haro Marín S, Orgiler Uranga PE, Vela Morales C, ¿Se respeta el sueño de los pacientes?. Rev Rol Enf 2001;24(7-8):555-58.

78.- Molano Álvarez E, Cornejo Bauer C, García Hernández R, Rojo Cabello S, Cuenca Solanas M, García Fuentes C. Enfermería de cuidados críticos y técnicas continuas de reemplazo renal en la Comunidad de Madrid. Enferm Intensiva 2003;14(4):135-47.

79.- Navarro JM, Orgiler PE, De Haro S. Valoración de los profesionales enfermeros del Informe de alta de Cuidados Intensivos. Metas 2004;7:6-10.

80.- Ania González N, Martínez Mingo A, Eseberri Sagardoy M, Margall Coscojuela $M^{a} A$, Asiain Erro $\mathrm{M}^{\mathrm{a}} \mathrm{C}$. Evaluación de la competencia práctica y de los conocimientos científicos de enfermeras de $\mathrm{UCl}$ en la aspiración endotraqueal de secreciones. Enferm Intensiva 2004;15(3):101-11.

81.- Montial Fernández E, Ruiz de Escudero García N, Prieto Arriba E. ¿Intuición o exactitud?. Posicionamiento intuitivo de la cabecera de la cama en pacientes críticos. ¿Necesitamos medirlo?. Enferm Intensiva 2005;16(2):54-61.

82.- Gutiérrez Alejandro A, Calvo Buey JA, Marcos Camina RMạ. Estudio para la disminución de errores en el registro de los balances hídricos de pacientes críticos ingresados en una unidad de cuidados intensivos. Enferm Intensiva 2005;16(3):100-09.

83.- Navarro-Arnedo JM, Orgiler-Uranga PE, de Haro-Marín S. Nuevo informe de alta de enfermería de cuidados intensivos: grado de satisfacción y cumplimentación de los colectivos implicados. Enferm Clin 2006;16(4):190-7.

84.- Rodríguez Montero Mạa $\mathrm{C}$, Rubio Hontanilla N. Manejo del dolor en relación a la experiencia del profesional. Tribuna Sanitaria 2007;marzo(201):19-20.

85.- Quesada Ramos C, García Díez R. Evaluación del grado de conocimiento de las recomendaciones para la prevención y el cuidado de úlceras por presión en Unidades Críticas. Enferm Intensiva 2008;19(1):23-34.

86.- González Sánchez JA, Cosgaya García O, Simón García Mã J, Blesa Malpica AL. Registros de enfermería: convencional frente a informatizado. Unidad de cuidados críticos. Enferm Intensiva 2004;15(2):53-62.

87.- García Jiménez A, Feal Fonte D, Miralles Frutos C, Barcia Piñeiro M ${ }^{a}$, Goiriz Montero D. Evaluación del aplicativo infomático Gacela ${ }^{\circledR}$ por el personal sanitario de una unidad de cuidados intensivos. Enfermería Cientifica 2005;276-277:80-85.

88.- Navarro Arnedo JM, Orgiler Uranga PE, de Haro Marín S. AURORA 2003®: gestor de informes de alta de enfermería de cuidados intensivos. Enferm Intensiva 2007;18(2):78-89.

89.- Navarro arnedo JM. Análisis de las páginas web de las Unidades de Cuidados Intensivos de España. Enferm Intensiva 2009;20(4):148-58.

90.- Velasco Bueno JM, Prieto de Paula JF, Castillo Morales J, Merino Nogales N, PereaMilla López E. Organización de las visitas de familiares en las unidades de cuidados intensivos de España. Enferm Intensiva 2005;16(2):73-83.

91.- Rodríguez Mondéjar JJ, Cevidanes Lara MM, Herrera Romero T, Rodríguez Soler J, López Amorós A, Peñalver Hernández F, et al. Análisis de las comunicaciones admitidas en los congresos nacionales de la SEEIUC en 8 años (1998-2005). Enferm Intensiva 2008;19(4):179-92.

92.- García-Labattut A, Tena F, Díaz J, Pajares R, Sandoval J, González J et al. Nivel de conocimiento sobre las Unidades de Cuidados Intensivos (UCI) y los médicos intensivistas entre los ciudadanos de Castilla y León. Med Intensiva 2006;30:45-51.

93.- Santana Cabrera L, Falcón Moreno R, Pérez Sánchez R, González González M. Percepción del personal de enfermería de hospitalización acerca de los cuidados intensivos. Enferm Intensiva 2009;20:127. 
94.- Williams G, Chaboyer W, Thornsteindóttir R, Fulbrook P, Shelton C, Chan D et al. Worldwide overview of critical care nursing organizations and their activities. International Nursing Review 2001;48(4):208-17.

95.- Marshall A. Research priorities for australian critical care nurses: do we need them?. Australian Critical Care 2004;17(4):142-50.

96.- Marín Fernández B. Prioridades en la investigación de enfermería en las unidades de cuidados intensivos. Enferm Intensiva 1994;5(1):23-27.

97.- Almendros Corral L, Bosch Artigues M, Ferrer Pujol E, Gutierrez Carrasco J, Solano Soria J, Abizanda R. Medicina Intensiva. Cálculo de plantillas de enfermería. Rev Rol Enf 1986; IX(93):13-16.

98.- Barroso Díaz A, Fuente Juárez AI, López Cid JJ, Millán Vázquez FJ, Rosado Muñoz N, Simón García MJ et al. Análisis de uso de los recursos humanos enfermeros en una unidad de cuidados intensivos polivalente. Situación con el resto de UCls europeas. Enferm Intensiva 2001;12(3):127-34.

99.- Muñoz Camargo JC. ¿Se debería legislar la obligatoriedad de ratios mínimos Enfermera/Paciente?. Enferm Intensiva 2006;17:139-40.

100.- Bray K, Wren I, Baldwin A, St Ledger U, Gibson V, Goodman S et al. Standards for nurse staffing in critical care units determined by: The British Association of Critical Care Nurses, The Critical Care Networks National Nurse Leads, Royal College of Nursing Critical Care and In-flight Forum. Nurs Crit Care 2010;15(3):109-11.

101.- Penoyer DA. Nurse staffing and patient outcomes in critical care: a concise review. Crit Care Med 2010;38(7):1521-8.

102.- Zaforteza Lallemand C, García Mozo A, Quintana Carbonero R, Sánchez Calvín C, Abadía Ortiz E, Miró Gayà JE. Abrir la unidad de cuidados intensivos a los familiares: ¿qué opinan los profesionales?. Enferm Intensiva 2010;21:52-7.

103.- El Hospital de Valme pilota la efectividad de la implicación familiar en el proceso de extubación del paciente crítico. Diario Enfermero 2010 [serie en Internet] no:394. Disponible en: http://www.enfermundi.com/boletincge/num394.htm [consultado el 20 de junio de 2010] 104.- Santana Cabrera L, Sánchez-Palacios M. La presencia del familiar en el momento del fallecimiento mejora la calidad del morir en la unidad de cuidados intensivos. Enferm Intensiva 2010;21:83-4.

ISSN 1695-6141

@) COPYRIGHT Servicio de Publicaciones - Universidad de Murcia 